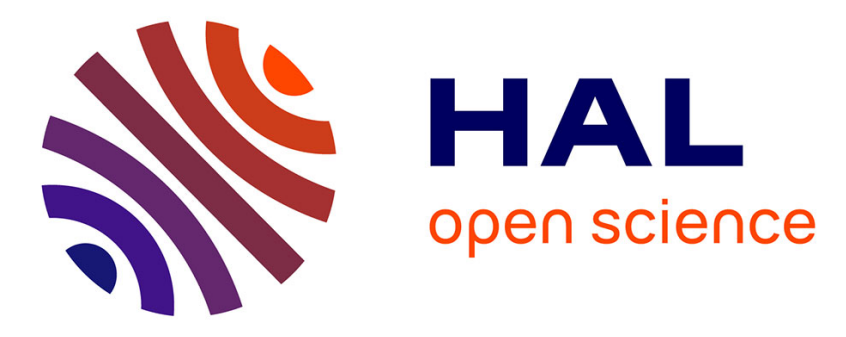

\title{
Solid-state dual-frequency laser free from anti-phase noise
}

\author{
Abdelkrim El Amili, Goulc'Hen Loas, Syamsundar De, Sylvain Schwartz, \\ Gilles Feugnet, Jean-Paul Pocholle, Fabien Bretenaker, Mehdi Alouini
}

\section{To cite this version:}

Abdelkrim El Amili, Goulc'Hen Loas, Syamsundar De, Sylvain Schwartz, Gilles Feugnet, et al.. Solidstate dual-frequency laser free from anti-phase noise. Conference on Lasers and Electro-Optics International Quantum Electronics Conference, May 2013, Munich, Germany. pp.CA_10_6. hal01044910

\author{
HAL Id: hal-01044910 \\ https://hal.science/hal-01044910
}

Submitted on 24 Jul 2014

HAL is a multi-disciplinary open access archive for the deposit and dissemination of scientific research documents, whether they are published or not. The documents may come from teaching and research institutions in France or abroad, or from public or private research centers.
L'archive ouverte pluridisciplinaire HAL, est destinée au dépôt et à la diffusion de documents scientifiques de niveau recherche, publiés ou non, émanant des établissements d'enseignement et de recherche français ou étrangers, des laboratoires publics ou privés. 


\title{
Solid-state dual-frequency laser free from anti-phase noise
}

\author{
A. El Amili', G. Loas' ${ }^{\text {, S. Se }}{ }^{2}$, S. Schwartz ${ }^{3}$, G. Feugnet ${ }^{3}$, J.-P. Pocholle ${ }^{3}$, F. Bretenaker ${ }^{2}$, M. Alouini, ${ }^{\text {I, }}$ \\ 1. Institut de Physique de Rennes, Université de Rennes 1, CNRS, Campus de Beaulieu, 35042 Rennes, France \\ 2. Laboratoire Aimé-Cotton, CNRS-Université Paris 11, 91405 Orsay Cedex, France \\ 3. Thales Research and Technology, RD 128, 91767 Palaiseau Cedex, France
}

Dual-frequency solid-state lasers are attractive for a large number of applications such as microwave photonics, spectroscopy, and metrology. As far as dual-frequency lasers are considered, the intensity noise spectrum of each eigenmode exhibits two peaks lying at the well-known in-phase and anti-phase eigenfrequencies of two coupled oscillators. The in-phase noise, which corresponds to the standard relaxation oscillations of the laser, can be reduced either electronically or optically using feedback loops [1]. However, the anti-phase noise, which is related to a resonant exchange of energy between the two laser modes, is very difficult to circumvent [2] because the reduction of this noise would require additional servo-loop acting on the difference of the intensities of the two modes or two servo-loops acting independently on the intensity of each mode. An optimal dual-frequency laser in terms of intensity noise and beat frequency stability would be a single axis laser in which the population inversions related to each mode are independent. We experimentally demonstrate how the proper design of a two polarization dual-frequency solid-state laser allows to get rid of the anti-phase noise in the simplest possible architecture and without using any electronic or optical feedback loop. This design is based on an appropriate choice of the active medium cut and orientation in order to assign two almost independent families of active atoms to the two laser modes.
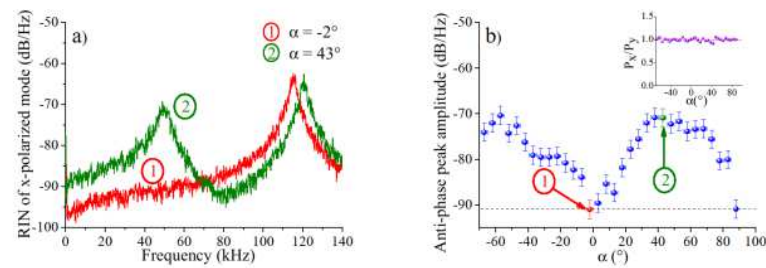

Fig. 1 RIN spectra for the x-polarized mode only for different values of $\alpha$. b) Evolution of the amplitude of the antiphase peak versus $\alpha$. Dashed line: noise floor level below which the anti-phase peak is no longer measurable. The inset shows the evolution of the intensity ratio of two orthogonal modes versus $\alpha$.

Besides, it has recently been shown that, in Nd:YAG, the emitting dipoles behave as if they were aligned along the crystallographic axes of the matrix [3]. In particular, by choosing a (100)-cut Nd:YAG crystal instead of the more common (111) cut, it was shown that almost complete decoupling of two perpendicularly polarized modes could be obtained by aligning them with the (010) and (001) crystallographic axes. One then expect the antiphase noise to vanish using a properly oriented (100)-cut Nd:YAG crystal. In order to adjust the angle, $\alpha$, between the laser polarization eigen-states and the crystallographic axes of the active medium, we insert into the two-mirror cavity a birefringent plate (BP) whose neutral axes define the two crossed and linear polarizations of the laser. Fig. 1(a) reproduces the relative intensity noise (RIN) spectra recorded when only the x-polarized mode is detected for two values of angle $\alpha$ corresponding to the two situations where the eigen-polarizations of the laser are (1) aligned with the crystallographic axes of the active medium $\left(\alpha=-2^{\circ}\right)$ or $(2)$ at $45^{\circ}\left(\alpha=43^{\circ}\right)$. We observe the existence of the usual in-phase relaxation oscillations peak at about $115 \mathrm{kHz}$ and $120 \mathrm{kHz}$. When $\alpha$ is set at $43^{\circ}$, that is the angle for which the coupling is maximum, the amplitude of the anti-phase peak is found to be maximum. By contrast, if we now rotate $\alpha$ and look for the position in which the amplitude of the anti-phase peak is minimized, we find $\alpha=-2^{\circ}$ as shown by the red spectrum of Fig. 1(a). The evolution of the anti-phase peak amplitude versus $\alpha$ is plotted in Fig. 1 (b). Consequently, we show that the noise induced by the anti-phase relaxation oscillations resonance in a dual-frequency laser can be almost completely cancelled by a proper choice the active medium and design of the cavity. This has been illustrated in the case of a Nd:YAG crystal in which the choice of a (100)-cut crystal together with the proper orientation of the polarizations of the laser modes permit to cancel this resonance by more than $20 \mathrm{~dB}$ [4].

\section{References}

[1] S. Taccheo, P. Laporta, O. Svelto, and G. de Geronimo, "Intensity noise reduction in a single-frequency ytterbium-codoped erbium laser," Opt. Lett. 21, 1747-1749 (1996).

[2] G. Pillet, L. Morvan, M. Brunel, F. Bretenaker, D. Dolfi, M. Vallet, J. P. Huignard, and A. Le Floch, "Dual-frequency laser at $1.5 \mu \mathrm{m}$ for optical distribution and generation of high-purity microwave signals," J. Lightwave. Technol. 26, 2764-2773 (2008).

[3] S. Schwartz, G. Feugnet, M. Rebut, F. Bretenaker, and J. P. Pocholle, " Orientation of $\mathrm{Nd}^{3+}$ dipoles in yttrium aluminum garnet: experiment and model," Phys. Rev. A 79, 063814 (2009).

[4] A. El Amili, G. Loas, S. De, S. Schwartz, G. Feugnet, J. P. Pocholle, F. Bretenaker, and M. Alouini, "Experimental demonstration of a dual-frequency laser free from antiphase noise," Opt. Lett. 37, 4901-4903 (2012). 